\title{
Nailfold capillaroscopic findings in a semi-professional volleyball player
}

\author{
Maria Maddalena Sirufo, Lia Ginaldi and Massimo De Martinis* \\ Department of Life, Health, and Environmental Sciences, University of L'Aquila, L'Aquila, Italy
}

\begin{abstract}
Volleyball players are exposed to the possibility of several well recognized injuries, among which microvascular abnormalities are the less described and studied, although they could diminish their ability and performance. Capillaroscopy is a simple, non-invasive method to diagnose vascular abnormalities in athletes suffering for the consequences of repeated application of pressure by local trauma on the fingertips. The detected capillaroscopic picture will be the base for the follow-up and will indicate the possible need for further investigations aimed at excluding other occulted conditions. At our knowledge, this is the first report describing and documenting the related microvascular abnormalities in a volleyball player. This case emphasizes the fact that volleyball players among other athletes, whose hands are exposed to repeated fingertips trauma may suffer from microcirculation damage, for which reason they should be able to access an effective health surveillance program able to detect the first signs and offer the most appropriate clinical support.
\end{abstract}

Keywords: Volleyball, athletes, sport, capillaroscopy, hand, injury, microcirculation, translational medicine

\section{Introduction}

Elite athletes are exposed to the possibility of a variety of injuries and although musculoskeletal lesions are the most common cause of complain [1], some of the most devious and disabling could be vascular injuries [2, 3]. The causes of venous or arterial injuries in athletes could be high-speed collisions and repetitive motions. Symptoms could be striking, or on the contrary, blurred. In most cases athletes are healthy and young and vascular diseases could be overlooked [4].

Volleyball, that is estimated to have 500 million players all over the world [5], is a discipline with an high prevalence of ischemia related symptoms. In the Netherlands one third of male volleyball players reported pale and/or blue fingers and symptoms of cold. Most literature on the topic refer to vascular conditions that sometimes could have a rapid and sudden course with catastrofic consequences [6-8]. Volleyball produces the impact of a ball on fingertips, which is repeated many many times during training and competitions that became thousands of times throughout an entire sporting career and similar to Basque handball game could produce Raynaud syndrome and related microvascular abnormalities [9]. Local arterial injury and thrombosis is described in football and baseball players due to repeated application of pressure by local trauma to a digital artery [4].

Frequently volleyball players complain of discomfort in their hands attributable to microvascular abnormalities such as cold hands. This aspect is often overlooked and there are no significant data in this regard in the literature. Disturbances can occur in a single digit or affect all the fingers simultaneously, it can be bilateral or unilateral. Microvascular symptoms can be the consequences of several different conditions or causes, including connective tissue diseases such as systemic sclerosis, extrinsic

\footnotetext{
${ }^{*}$ Corresponding author: Massimo De Martinis, Department of Life, Health, and Environmental Sciences, University of L'Aquila, Piazzale Salvatore Tommasi, L’Aquila, 67100, Italy. E-mail: demartinis@cc.univaq.it.
} 
vascular obstruction, certain chemicals/drugs, traumatic injuries, vibration exposure (hand-arm vibration syndrome), hyperviscosity states (paraproteinaemia) and anorexia nervosa [10-12].

\section{Case report}

We refer about a 17-year-old woman, volleyball player, visited for purple, swollen and sweating hands when playing that persists for a few hours after the end of the game, predominantly in the right hand. This happens in the last two years and it is worsening.

She had no history of diabetes mellitus, cardiovascular or neurologic disease, thyroid disease, arthritis, or connective tissue disease, no history of carpal tunnel syndrome or frostbite, no family history of primary Raynaud phenomenon or connective tissue disease. She was not taking any medications and she is right-hander.

She doesn't smoke. She play volleyball an average of three hours a day four days a week, since she was twelve years old.

The physical examination was unrewarding. She had normal vital signs and no remarkable findings on cardiovascular, neurologic, and musculoskeletal examinations. In particular a careful clinical examination and laboratory investigations excluded known pathologies that could lead to nailfold microvascular abnormalities. She did not have symptoms or established diagnosis of connective tissue diseases, neither presented other known causes able to determine NVC abnormalities (no digital ulcers or skin manifestations suggesting a diagnosis of SLE or scleroderma, or suffering from muscle and joint pain). We performed nailfold videocapillaroscopy (NVC) to assess microcirculatory alteration in our young athlete $[13,14]$.

NVC was performed by using a x200 optical probe equipped videocapillaroscope connected to an image analyzer software (VideoCap software 3.0; DS Medica, Milan, Italy). After a 15 min. adaptation at room temperature of $23^{\circ} \mathrm{C}$, a drop of immersion oil was applied to the nailfolds of the second, third, fourth and fifth fingers of both hands in order to increase transparency of the keratin layer, and all of them were examined in each patient.

We analyzed skin transparency, capillary density (normally between 9 to 14 homogenously distributed hairpin-shaped capillaries in $1 \mathrm{~mm}$ ), ramified capillaries and tortuosities (single or multiple crossovers) [15], microhaemorrhages [16] and disorganization of the vascular array. We also evaluated the presence of enlarged (irregular or homogeneous increase of capillary diameter $>20$ and $<50 \mathrm{~mm}$ ) and giant capillaries (homogeneously enlarged loop with a diameter $>50 \mathrm{~mm}$ ).

We found a lack of morphological homogeneity of capillaries, presence of ectasia of the efferent tract of the loops, apical ectasia, tortuosity, newly formed capillaries with bushy, branching and "candelabrum" appearance (Fig. 1); a "non-specific abnormal NVC pattern" without findings suggestive of scleroderma.

At our knowledge, this is the first report describing and documenting the related microvascular abnormalities in a volleyball player.

This work was conducted after receiving the patient's informed consent to participate to the study and to publish this report, in compliance with the ethical standards in the field and the norms esthablished by the Internal Review Board of our Institution.

\section{Discussion}

The volleyball player could suffer for the consequences of various injuries: the most frequent being musculoskeletal $[1,17]$. Vascular complications are reported, influenced by exposition to high-speed collision and repetitive motion and appear that arterial and venous abnormalities are becaming an 


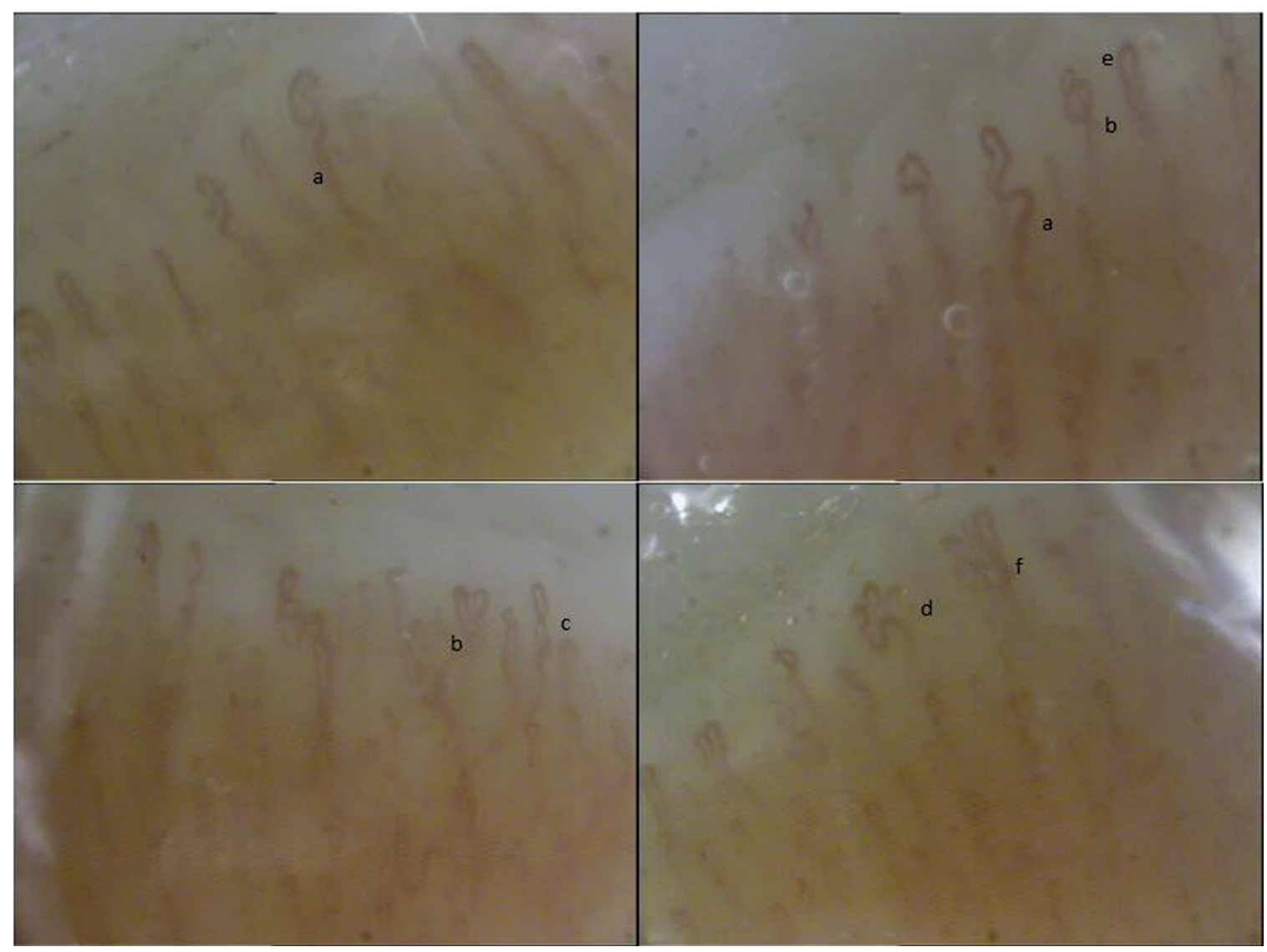

Fig. 1. Nailfold videocapillaroscopy images in a case of volleyball player showing a "non-specific pattern": a) ectasia of the efferent tract of the loops, b) apical ecatsia, c) tortuosity and newly formed capillaries with d1) bushy, d2) branching, d3) "candelabrum" appearance.

increasing problem in these athletes. Although severe vascular complication such as aneurysmal degeneration, thrombosis and distal occlusion of the proximal posterior circumflex humeral artery (PCHA) or Hypothenar or thenar hammer syndrome (HAVS) have been topic of previous studies $[8,18,19]$ and are well recognized in these patients, microvascular anomalies, are less described and studied. Extremity pain, cold, blu and pale digits are signs associated to vascular disturbances that in athletes necessitate a careful evaluation. A vascular disease left undiagnosed for a prolonged interval, could have devastating consequences. After excluding more severe diseases the NVC allow in a simple non-invasive way to evaluate cutaneous microcirculation and diagnose the digital vascular abnormalities due to repeated fingertips trauma. NVC is a widely used test to diagnose digital vascular abnormalities in hand-arm vibration syndrome (HAVS) and differentiate from a secondary form depending by autoimmune/connective diseases such as scleroderma. Repeated traumas are a potential risk for microcirculation with an increased tendency to vasospasm in the digital capillaries. Volleyball involves low-to-high-velocity collisions between the ball and a player, the volleyball behaviour is unique for each different type and the interaction between the player and the ball depend on the ball type and the incident velocity. In collision injuries, the varying combinations of kinetic energy lost and peak impact force may be useful to understand the role of each of these variables [20]. In general, little is known about how the collision in itself is harmful and the question becomes more complicated about the athletes. Furthermore kinetic, force, intensity, duration, frequency of exposure and several 
other cofactors regulate dangerousness and should be taken into account. Age, sex and smoking may also influence the risk of developing microvascular disturbances.

\section{Perspective}

Currently NVC appear to be an accurate, reproducible, reliable and objective method to diagnose microvascular abnormalities, too often overlooked, in athletes and in volleyball players in particular. The capillaroscopic pattern will be the base for the follow up of the patient and will suggest if further investigations are needed to exclude other occulted pathologies such as connective tissue diseases. The detection of objective and documented vascular alterations, will help the athlete to achieve awareness of the condition, to reassure her/him while understanding the need for a follow-up and where possible for modifying some lifestyles and ways of playing. This to preserve health over time and to help control and, if possible, change factors that could affect the good athletic performance.

\section{Conflict of interest}

The authors have no conflict of interest to report.

\section{References}

[1] Kilic O, Maas M, Verhagen E, Zwerver J, Gouttebarge V. Incidence, aetiology and prevention of musculoskeletal injuries in volleyball: A systematic review of the literature. Eur J Sport Sci. 2017;17(6):765-93. doi: 10.1080/17461391. 2017.1306114

[2] Arko FR, Harris EJ, Zarins CK, Olcott C 4th. Vascular complications in high-performance athletes. J Vasc Surg. 2001;33(5):935-42.

[3] Wahl U, Kaden I, Köhler A, Hirsch T. Vascular trauma of the hand - a systematic review. Vasa. 2019;48(3):205-15. doi: 10.1024/0301-1526/a000743

[4] Perlowski AA, Jaff MR. Vascular disorders in athletes. Vasc Med. 2010;15(6):469-79. doi: 10.1177/1358863X10382944

[5] Van De Pol D, Alaeikhanehshir S, Maas M, Kuijer PP. Self-reported symptoms and risk factors for digital ischaemia among international world-class beach volleyball players. J Sports Sci. 2016;34(12):1141-7. doi: 10.1080/02640414. 2015.1093649 Epub 2015 Oct 5.

[6] Trommeter RA, Freeman CL, Shah KS, McKinzie JP, Smith AT. Hypothenar hammer syndrome. J Emerg Med. 2019;56(1):105-6. doi: 10.1016/j.jemermed.2018.09.042 Epub 2018 Nov 9

[7] Kasitinon D, Dimeff RJ. Dropping the hammer: An uncommon case of right-hand pain in a professional hockey player. Sports Health. 2018:1941738118816050. doi: 10.1177/1941738118816050

[8] van de Pol D, Kuijer PPFM, Terpstra A, Pannekoek-Hekman M, Alaeikhanehshir S, Bouwmeester O, Planken RN, Maas M. Posterior circumflex humeral artery pathology and digital ischemia in elite volleyball: Symptoms, risk factors \& suggestions for clinical management. J Sci Med Sport. 2018;21(10):1032-7. doi: 10.1016/j.jsams.2018.03.010

[9] Letamendia A, Lòpez-Román J, Bustamante-Munguira J, Herreros J. Digital periarterial sympathectomy in the management of post-traumatic Raynaud syndrome. J Vasc Surg. 2016;63(2):459-65. doi: 10.1016/j.jvs.2015.08.102

[10] De Martinis M, Ciccarelli F, Sirufo MM, Ginaldi L. An overview of environmental risk factors in systemic sclerosis. Exp Rev Clin Immunol. 2016;12(4):465-78. doi: 10.1586/1744666X.2016.1125782

[11] De Martinis M, Sirufo MM, Ginaldi L. Raynaud's phenomenon and nailfold capillaroscopic findings in anorexia nervosa. Curr Med Res Opin. 2018:1-4. doi: 10.1080/03007995.2017.1417828

[12] Sirufo MM, Ginaldi L, De Martinis M. Raynaud's phenomenon and the nailfold capillaroscopic findings in a guitar player. QJM: An International Journal of Medicine. 2019:hcz095, https://doi.org/10.1093/qjmed/hcz095

[13] Jung F, Wappler M, Nüttgens HP, Kiesewetter H, Wolf S, Müller G. Method of video capillary microscopy: Determination of geometric and dynamic measuring parameters. Biomed Tech (Berl). 1987;32(9):204-13.

[14] Fagrell B, Hermansson IL, Karlander SG, Ostergren J. Vital capillary microscopy for assessment of skin viability and microangiopathy in patients with diabetes mellitus. Acta Med Scand Suppl. 1984;687:25-8. 
[15] Koscielny JK, Latza R, Mürsdorf S, Mrowietz C, Kiesewetter H, Wenzel E, Jung F. Capillary microscopic and rheological dimensions for the diagnosis of von Willebrand disease in comparison to other haemorrhagic diatheses. Thromb Haemost. 2000;84(6):981-8.

[16] Leithäuser B, Mrowietz C, Hiebl B, Pindur G, Jung F. Capillary bleeding under oral anticoagulation. Clin Hemorheol Microcirc. 2009;43(1-2):167-71.

[17] Rosenbaum YA, Awan HM. Acute hand injuries in athletes. Phys Sportsmed. 2017;45(2):151-8. doi: 10.1080/00913847. 2017.1306420

[18] van de Pol D, Kuijer PP, Langenhorst T, Maas M. Risk factors associated with self-reported symptoms of digital ischemia in elite male volleyball players in the Netherlands. Scand J Med Sci Sports. 2014;24(4):e230-7. doi: 10.1111/ sms. 12145

[19] van de Pol D, Kuijer PP, Langenhorst T, Maas M. High prevalence of self-reported symptoms of digital ischemia in elite male volleyball players in the Netherlands: A cross-sectional national survey. Am J Sports Med. 2012;40(10):2296-302.

[20] Chiu LZF, vonGaza GL. Analysis of different volleyballs' collision mechanics across a range of incident velocities. Sports Biomech. 2018:1-14. doi: 10.1080/14763141.2018.1535618 